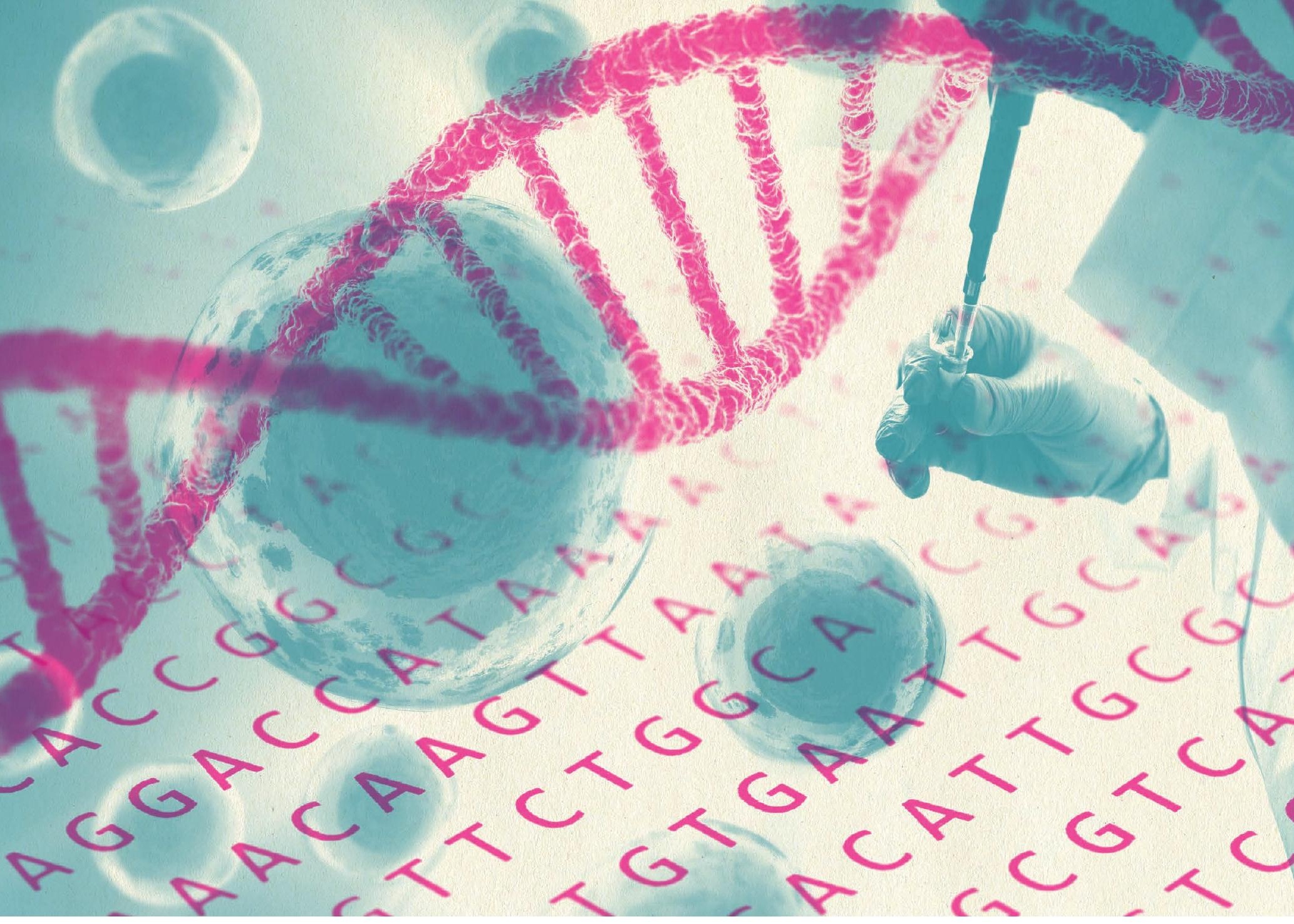

\title{
The genetic microscope
}

\section{Single-cell genomic sequencing is poised to revolutionize fields from cancer to immunology.}

\author{
BY PAUL SMAGLIK
}

\begin{abstract}
do Amit, a genome researcher and immunologist at the Weizmann Institute of Science in Rehovot, Israel, had a problem. When he and his colleagues were studying gene regulation in 2009, the genetic differences among the many different kinds of immune cells were hard to track, and conventional population-based sequencing provided only a limited view. To get a more specific picture, he needed something impossible at the time: genetic resolution at the single-cell level.
\end{abstract}

Since the mid-2000s, when the first commercial high-throughput (also known as nextgeneration) sequencers entered the market, the costs associated with digging into the genome have plummeted. As a result, the entire field has changed, opening up viable avenues into important topics such as personalized medicine and forensic science. High-throughput sequencing is invaluable, highly automated and extremely fast, but results in a composite view, rather than a specific picture, says Stephen Quake, a bioengineer at Stanford University in Palo Alto, California.

In addition, high-throughput genome sequencing relies on what Quake calls bulk tissue analysis. "You grind up the whole tissue, different cell types get averaged together and you get some average value for what's going on." This is good enough to view the genome of an entire organism, but it doesn't capture the often significant diversity - the heterogeneity - of genetic sequences among different cell types.

Single-cell genomic sequencing is different. "With single-cell genomics, you are focusing on each cell independently," says Quake, who was an early developer of the single-cell approach. As a result, the technique allows scientists to explore genetic diversity in organisms and systems, and to break down complicated biology into separate genetic data sets.

That approach was essential to the questions Amit was trying to answer about the immune system - a complicated place, with many types of cell all contributing to an overall biological process. Amit and his colleagues were one of a handful of groups to build single-cell techniques that have allowed scientists to dig deeper and see further into even subtle differences between individual cells on a genetic level. In short: scientists now have a genetic microscope, with resolution that goes all the way down to a single cell.

"This new 'microscope' opened up many basic research and biomedical directions we could not even dream of before in 
immunology," says Amit. "Within complex populations of cells, such as the immunetumour ecosystem, high-resolution-datadriven models can now be investigated, pinpointing specific cell types and pathways."

The caveat? Single-cell genomics is more technically and analytically challenging than conventional methods. Scientists need to extract the nucleic acids from one cell. They need to chemically amplify this sample millions of times while retaining the original genetic content. The informatics and analysis portions are also more challenging, because the technique can so far only capture a percentage of a single cell's transcripts, so the gaps must be filled in by sequencing and by using sophisticated computational methods.

\section{EVERY CELL IS DIFFERENT}

The promise of the technique far outweighs its challenges, says Charles Gawad, an oncologist at St Jude Children's Research Hospital in Memphis, Tennessee. As the approach becomes more widespread, it is helping scientists to understand the states of individual cells through efforts such as the Human Cell Atlas project (see 'A comprehensive catalogue of the body'), and aiding researchers who study particular diseases and systems that involve multiple cell types. It is especially useful in any instance when heterogeneity has a role.

This makes oncology a good fit for the technique, says Gawad. "We don't really know how heterogeneous tumours are," Gawad says. "We don't know how much drug resistance is already present within a tumour at the time of diagnosis."

His laboratory aims to survey tumours for this genetic diversity, clone by clone. In doing so, Gawad intends to reframe cancer not as a list of mutations available from a single tumour that contains many tissue types, but as a population-genetics problem. "This is not a new idea," he says. "But now we have new tools that can begin to deeply probe the genomics of a tumour, enabling the development of more quantitative models of a patient's cancer."

He also hopes to identify rare populations that harbour mutations that confer resistance to treatment. "The only way to define the problem is by using single-cell genomics to really deeply probe the genomics of the tumour," Gawad says. "We can begin to understand how those factors correlate with the outcome of the patient. We could then devise treatment strategies with greater precision that are based on the genomics of the tumour at cellular resolution."

He's pleased to be making use of this new generation of technology. "I'm doing something I feel very passionate about and feel longterm is going to have a real clinical impact." $\mathrm{He}$ encourages others interested in genetic heterogeneity in other fields - immunology, neurology and infectious diseases - to consider learning the technique even if it hasn't matured yet, saying it's the next paradigm shift in genomics, following next-generation sequencing. "If you got training early in nextgeneration sequencing, you were ahead of the curve."

\section{MINDFUL OF COMPUTATION}

Chris Walsh, a neuroscientist at Children's Hospital Boston and Harvard University in Cambridge, Massachusetts, has been wondering how the human brain develops since his graduate school days. Single-cell genomics is helping him to answer that question.

He uses two types of single-cell technique to understand neurogenesis. In one set of experiments, he sequences single-cell RNA as a way to characterize stem-cell types in a developing brain. In another set, he sequences the DNA of neurons in the adult brain to identify mutations shared by some - but not all - neurons from the human cerebral cortex, because these 'somatic' mutations can help to track the lineages of brain cells.

"There is a permanent mark in the human brain of where all the different cells came from," Walsh says. "We just have to do enough sequencing to try and decode as much as we can."

\section{NOW WE HAVE NEW TOOLS THAT CAN BEEIN TO DEEPLY PROBE THE GENOMICS OFA TUMOUR.}

That decoding - the computational analysis - of the process is by far the most difficult part of the equation. Walsh says that without the support of the Peter Park bioinformatics lab at Harvard, he would have difficulty deriving meaning from his data.

Computational biologists are turning to the challenges of single-cell data, which, in turn, are providing career opportunities. Stephen Clark, a postdoctoral researcher in Wolf Reik's lab at the Babraham Institute in Cambridge, UK, prefers developing new methods and techniques over asking specific scientific questions.

"It's easy to see where the opportunities are," Clark says. "You can figure out what is possible and what is the lowest hanging fruit." For example, the Reik lab had already combined single-cell DNA and RNA sequencing for one specific problem, so he tweaked the protocol to capture a few more cellular processes.

Those techniques and others he helped to develop are now being used in a number of projects that range from developmental biology in embryos to heterogeneity in ageing. $\mathrm{He}$ likes both the independence of the work and the fact that others depend on him for doing it. "I'm sort of on my own here - the guy inventing new techniques," he says. After Clark concludes his postdoc, he intends to maintain his focus on technology development, because he enjoys the work and senses that demand will remain high.

\section{EXCITING POSSIBILITIES}

For Reik too, diving into single-cell genomics started as a technical challenge to answer a scientific question. His lab studies epigenetic marks - chemical tags along DNA sequences, which guide proteins to act on or ignore specific genes. This differentiation between expressed and non-expressed DNA allows the body to make different types of cell with the same set of genetic information, something of particular relevance in embryonic development and ageing.

As Reik was already working with a relatively rare and small cell population, he thought it would be fun to narrow that population down to its smallest element. "I am already quite close to one, so from a technological perspective, it would be quite exciting to get to one cell," Reik told himself.

This kind of 'what if' thinking may well drive the future of the field, Reik says. For instance, currently, cells must be killed before they can be sequenced. But keeping the cell alive and sequencing it over time would provide a window into how cells respond to processes such as DNA methylation, which Reik uses as an epigenetic marker. "Time resolution is one of the next big challenges," he says. Combining single-cell sequencing with imaging to see the cell on a physical and genetic level simultaneously would also be "super exciting", says Reik.

Amit cautions that there are many technical hurdles left to clear before single-cell sequencing can be as ubiquitous as next-generation sequencing. But he emphasizes that researchers should think in terms of methodology — in other words, reframing the scientific questions they ask and finding new ways to probe those questions at a molecular level. "The whole design of experiments needs to be different," Amit says.

Early adopters of single-cell sequencing were on their own in terms of developing methods. Initially, just a handful of labs were pursuing the technology. The only way to learn then was by joining one of these labs, like Gawad did.

Orit Rozenblatt-Rosen, scientific director of the Klarman Cell Observatory of the Broad Institute in Cambridge, Massachusetts, and the lead scientist at the Broad for the International Human Cell Atlas Initiative, helped to 


\section{HUMAN CELL ATLAS \\ A comprehensive catalogue of the body}

The Human Genome Project was the most ambitious big-science effort of its time. But an effort to describe every single cell type of the human body may well dwarf it, as it will involve profiling many millions of individual cells. The Human Cell Atlas initiative kicked off in October last year at a meeting in London attended by researchers from around the world. It is being further supported by a call for pilot projects funded by the Chan Zuckerberg Initiative as part of a wider 'Biohub' initiative, which has a goal to "cure, prevent or manage all diseases".

Mike Stubbington, who leads a team working on the Human Cell Atlas at the Wellcome Trust Sanger Institute in Hinxton, UK, says that the initiative is the next frontier in biological research. Since the mid-seventeenth century, biologists have attempted to categorize cell types. This categorization has progressed from shape, to tissue to location and on to individual molecular components. "There isn't a deep systematic catalogue of cell types yet. The initiative will index cells by their gene expression profiles and ultimately their spatial positions, to create an atlas of human cells," Stubbington says.

The atlas project will create a complete cellular resource and establish best practices such as minimum standards for data. Members will develop and share new techniques in both experimental analysis and data processing. The effort's next meeting is on 1-2 June in Stockholm, but the atlas launch date has not yet been set. P.S. launch the observatory in 2012, when singlecell methods were first being developed and applied. "These new technologies were super exciting to me," she says.

She continues to work with collaborators at the Broad Institute and beyond to organize single-cell research initiatives. Doing so has enabled her to participate in the development and application of cutting-edge technologies and to learn the skills necessary to lead and navigate large collaborations. "One of our aims is to help scientists learn how to use single-cell genomics, so we do quite a lot of teaching and dissemination," she says. "We help them plan their experiments, we show them how to use the technology and help computationally analyse the results, we try to have as many tools in-house as possible - and when we need to, we co-invent technologies."

In her own research, Rozenblatt-Rosen uses single-cell sequencing to study tumour cell heterogeneity - she, like Gawad, wants to break tumours down into their component tissue parts. Today, she's especially interested in looking at differences in immune cells within tumour types, because only some cancers respond well to immunotherapy. Together with close collaborators at Massachusetts General Hospital and the Dana-Farber Cancer Institute in Boston, Massachusetts, she is working on building systematic pipelines from the hospitals to the Broad Institute to profile tumour samples from patients undergoing clinical care. "I'm very, very excited about embedding this process into the precision-medicine pipeline," she says.

\section{TRAINING SEQUENCE}

As the field matures, there are now more training opportunities, says Tanja Woyke of the US
Department of Energy's Joint Genome Institute (JGI) in Walnut Creek, California. Some labs host visiting scientists. Others offer talks. Still more provide workshops and courses.

The single-cell approach is "very complementary" to metagenomics, which looks collectively at the genome of many species living in a particular environment, Woyke says. For instance, one could use metagenomics to sequence all of the microbial bulk DNA in a handful of soil, then perform single-cell sequencing on a few organisms found in that sample and, finally, compare all of the sequencing data.

If someone has an interest in single-cell genomics but doesn't have the technical knowhow, the JGI can perform single-cell isolations, genome amplification and sequencing for them under the umbrella of the Community Science Program (go.nature.com/2ql2sth). There are some caveats, of course. The research problem must be relevant to the Department of Energy's areas of interest - bioenergy, carbon cycling and biogeochemistry - and the user must submit a proposal that will be peer reviewed, says Woyke.

The JGI now sequences thousands of singlecell genomes a year. "Demand is very high," says Woyke. She sees growth as inevitable, especially in microbiology, as only about $15 \%$ of known microorganisms can be grown in culture. The other $85 \%$ must be sequenced by single-cell methods. As the technology becomes cheaper and easier, which she sees as inevitable, single-cell genomics will be an important technique in every molecular biologist's toolbox, just as next-generation sequencing is today.

Paul Smaglik is a freelance writer in Milwaukee, Wisconsin. 
CORRECTION

The Spotlight article 'The genetic

microscope' (Nature 545, S25-S27; 2017)

said that Orit Rozenblatt-Rosen was an

associate director at the Klarman Cell

Observatory. In fact, she is the scientific

director there. 